\title{
Genome-wide association studies of yield- related traits in high-latitude japonica rice
}

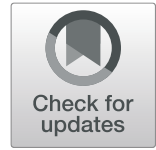

\author{
Guomin Zhang ${ }^{1,2+}$, Rongsheng Wang ${ }^{1,2 \dagger}$, Juntao Ma ${ }^{1,2}$, Hongru Gao ${ }^{1,2}$, Lingwei Deng ${ }^{1,2}$, Nanbo Wang ${ }^{1,2}$, \\ Yongli Wang ${ }^{1,2}$, Jun Zhang ${ }^{1,2}$, Kun $\mathrm{Li}^{1,2}$, Wei Zhang ${ }^{1,2}$, Fengchen Mu ${ }^{1,2}$, Hui Liu ${ }^{1,2}$ and Ying Wang ${ }^{1,2^{*}}$
}

\begin{abstract}
Background: Heilongjiang Province is a high-quality japonica rice cultivation area in China. One in ten bowls of Chinese rice is produced here. Increasing yield is one of the main aims of rice production in this area. However, yield is a complex quantitative trait composed of many factors. The purpose of this study was to determine how many genetic loci are associated with yield-related traits. Genome-wide association studies (GWAS) were performed on 450 accessions collected from northeast Asia, including Russia, Korea, Japan and Heilongjiang Province of China. These accessions consist of elite varieties and landraces introduced into Heilongjiang Province decade ago.

Results: After resequencing of the 450 accessions, 189,019 single nucleotide polymorphisms (SNPs) were used for association studies by two different models, a general linear model (GLM) and a mixed linear model (MLM), examining four traits: days to heading $(\mathrm{DH})$, plant height $(\mathrm{PH})$, panicle weight (PW) and tiller number (TI). Over 25 SNPs were found to be associated with each trait. Among them, 22 SNPs were selected to identify candidate genes, and 2, 8, 1 and 11 SNPs were found to be located in 3' UTR region, intron region, coding region and intergenic region, respectively.
\end{abstract}

Conclusions: All SNPs detected in this research may become candidates for further fine mapping and may be used in the molecular breeding of high-latitude rice.

Keywords: Oryza sativa japonica, GWAS, Yield trait, Resequencing, Rice breeding

\section{Background}

Rice cultivated in Asia is the staple food for most of the population worldwide. Research on its genetic variation, population structure and diversity has advanced greatly in recent decades [1-3]. Cultivated rice belongs to different subspecies or varietal groups and shows different domestication characteristics. Additionally, the domesticated subspecies include two main groups: Oryza sativa japonica and $O$. sativa indica. However, evidence suggests that they may have been domesticated separately

\footnotetext{
* Correspondence: wangyingqq2005@163.com

${ }^{\dagger}$ Guomin Zhang and Rongsheng Wang contributed equally to this work. 'Biotechnology Research Institute, Heilongjiang Academy of Agricultural Sciences, Harbin, China

${ }^{2}$ Northern Japonica Rice Molecular Breeding Joint Research Center, Chinese Academy of Sciences, Harbin, China
}

from the ancestral species approximately 18 and 12 thousand years ago [4]. Genomic studies have confirmed the differentiation of three subspecies within O. sativa japonica, temperate, subtropical and tropical japonica, which grow in diverse environments with different climate characteristics [5].

Every subspecies may have distinctive signatures or alleles that are formed during domestication or artificial selection. People in a specific area selected particular traits for their consumption needs [6]. Many studies of different genes showed clear evidence of positive selection during the evolutionary process, such as genes related to waxiness and cold tolerance [7-9]. Research focused on a subspecies or a population collected from a specific geographical region may reveal distinctive

(c) The Author(s). 2021 Open Access This article is licensed under a Creative Commons Attribution 4.0 International License, which permits use, sharing, adaptation, distribution and reproduction in any medium or format, as long as you give appropriate credit to the original author(s) and the source, provide a link to the Creative Commons licence, and indicate if changes were made. The images or other third party material in this article are included in the article's Creative Commons licence, unless indicated otherwise in a credit line to the material. If material is not included in the article's Creative Commons licence and your intended use is not permitted by statutory regulation or exceeds the permitted use, you will need to obtain permission directly from the copyright holder. To view a copy of this licence, visit http://creativecommons.org/licenses/by/4.0/ The Creative Commons Public Domain Dedication waiver (http://creativecommons.org/publicdomain/zero/1.0/) applies to the data made available in this article, unless otherwise stated in a credit line to the data. 
characteristics. Moreover, functional alleles or loci will be identified with certain analysis methods.

Quantitative trait locus (QTL) analysis has turned out to be a very effective tool for gene and locus discovery in recent years. A large number of genes have been cloned based on QTLs from different species around the world [10-12]. The emergence of high-throughput genome sequencing technology has decreased the expense and enhanced its efficiency. Combined with population phenotypes, many statistical analysis measures have been developed based on next-generation sequencing or single-nucleotide polymorphism (SNP) chips. This approach is called genome-wide association study (GWAS) and became widely used within a short time after being proposed. Its three main advantages over other population analysis methods are higher mapping resolution, a larger allele number and broader reference population, and lower time consumption [13]. Genome-wide association studies can be performed in a wide range of populations, such as germplasm resource material, $[14,15] \mathrm{F} 2$ populations, [16] nested association mapping (NAM) populations, [17] multiple advanced generation intercross (MAGIC) populations $[18,19]$ and random openparent association mapping (ROAM) populations [20, 21]. Multiple statistical models can be used in GWAS based on different populations or scales of SNP numbers, $[22,23]$ and population structure and genetic relationships can be taken into consideration [24, 25]. It has been more than 10 years since GWAS was first proposed, [26] and many mature workflows and analysis tools have been developed [27, 28]. The application of GWAS in rice has been widely reported in recent years. Alleles or SNPs located by GWAS have been applied in rice molecular breeding $[29,30]$.

Yield is one of the main traits that rice breeders focus on because of its relevance to worldwide food security. Nearly half of the world population consumes rice as a staple food. Yield is also known to be a multigene controlled trait, and many genes and loci have been found that could account for yield differentiation. Furthermore, yield is a complicated trait that is affected by many other traits, such as the tiller number, plant height, grain number, grain weight and number of primary branches [31, 32]. Association analysis with different populations may identify some unique genes or loci that contribute to specific traits. Therefore, it is necessary to identify novel genes or loci in a different population that may play a role only in a specific environment.

Rice cultivated at high latitudes in Asia shows many good characteristics, such as cold tolerance and high quality. There might be a large number of effective alleles that would be useful for further breeding for these traits. Few studies have focused on high-latitude natural populations and their effective alleles. In this research, we collected hundreds of cultivars and landraces as a natural population from high-latitude areas, including Northeast China, South Korea, Russia and Japan, and performed GWASs to examine their PH, PW, TI and $\mathrm{DH}$ in four different environments in Heilongjiang Province, China, with the aim of discovering genes or associated SNPs that could account for the differentiation of phenotypes and are expected to be used in further breeding programs.

\section{Results \\ Phenotyping of different traits}

The plant height of 450 accessions ranged from 51.33 $\mathrm{cm}$ to $146.67 \mathrm{~cm}$, with an average of $93.67 \mathrm{~cm}$ (Supplementary Table 2). In all four locations, plant height showed a normal distribution and a similar median with no significant difference except in Heihe, which may be due to a short growing season (Fig. 1A). The TI and PW in the four locations showed differences from each other, and in Heihe, these two traits showed narrower ranges than in the other locations (Fig. 1B-C). The DH showed a gradient change with increasing latitude. Wuchang and Harbin have similar means of DH, 94 and 93, respectively. However, at higher latitudes, DH significantly increased because of the longer daylight (Fig. 1D). Analysis of variance for the four traits showed significant differences $(<0.001)$. However, broad-sense heritability gave a higher estimate for $\mathrm{PH}(0.89)$ and $\mathrm{DH}(0.95)$ and a lower estimate for TI (0.68) and PW (0.47).

\section{Resequencing results and SNP distribution}

Approximately 1227 billion bases in total were obtained after resequencing, with an average of $6.3 \times$ sequencing depth and 18.9 million reads for each accession (Supplementary Table 1). Over 6.4 million SNP loci were called among the 450 accessions, indicating an average of 57 bp between pairs of SNPs. SNPs on each chromosome ranged from 0.39 million (chromosome 3) to 0.76 million (chromosome 11), with an average of 0.54 million per chromosome. According to the minor allele frequency (MAF) statistics, nearly $65 \%$ had an MAF less than $0.05,20.6 \%$ had an MAF greater than 0.1 and $8.1 \%$ had an MAF greater than 0.25 . The mean MAF on each chromosome varied between 0.052 and 0.085 , with an average of 0.07 across all chromosomes.

\section{Linkage disequilibrium and SNP distribution}

After filtering by MAF and missing genotype rate, 1,991,545 SNPs remained, and all these SNPs were used for linkage disequilibrium (LD) decay analysis across all chromosomes. The LD decay distance ranged from 15 $\mathrm{kb}$ to $27 \mathrm{~kb}$, with an average of $20 \mathrm{~kb}$ in the four groups predicted by population structure analysis (Supplementary Fig. 1A). However, the LD decay distance of all 


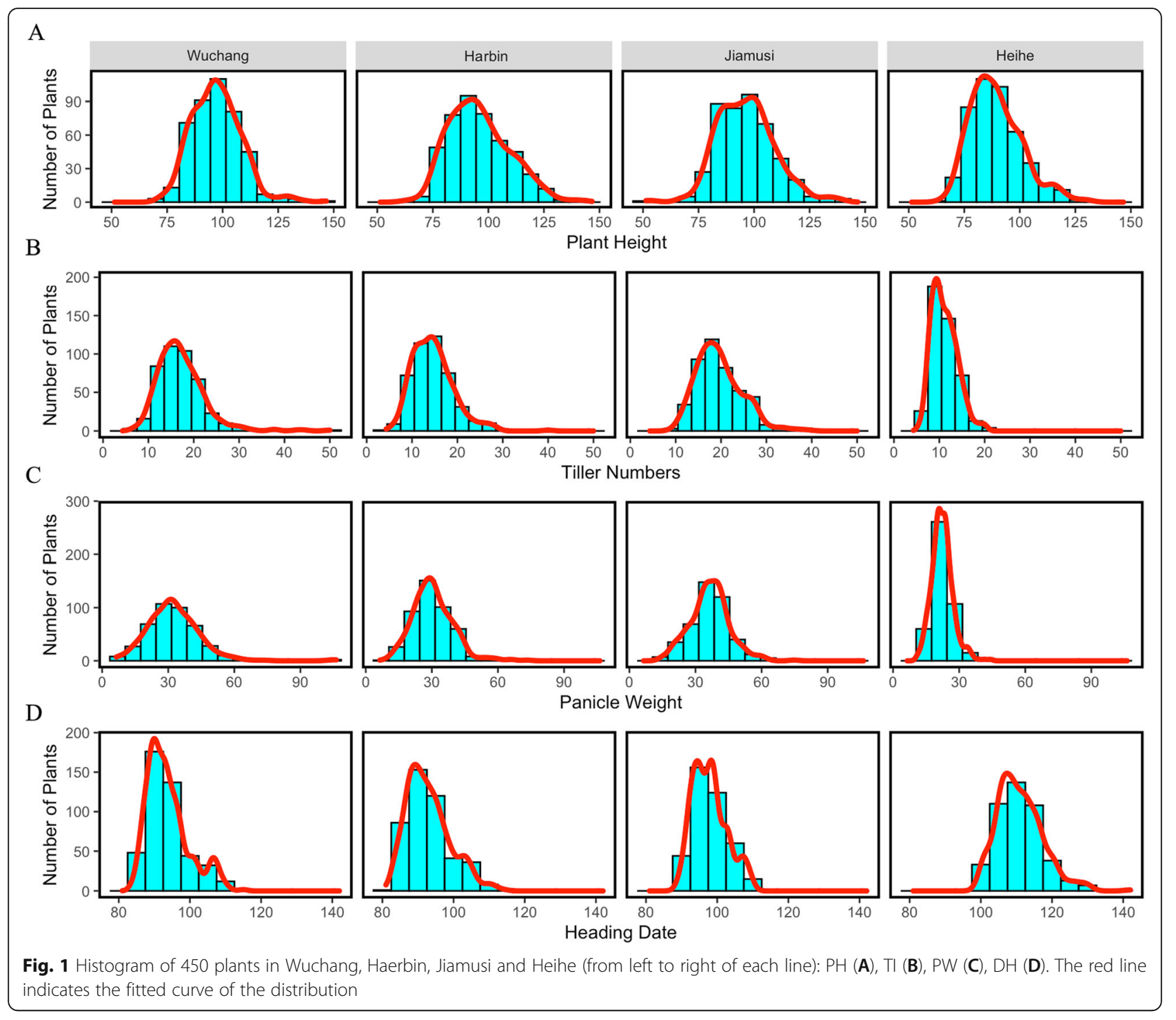

accessions was $23 \mathrm{~kb}$, which means that $r^{2}$ dropped to half of its maximum value (Supplementary Fig. 1B). This LD decay distance was lower than the previous estimate in temperate japonica but higher than that in O. rufipogon, [33] which may be affected by landraces in the population and possibly by having undergone weakened artificial selection. Finally, we filtered the SNPs based on the $r^{2}$ value, and 189,019 SNPs were retained for subsequent analyses, indicating one SNP every $2 \mathrm{~kb}$ across the whole genome. These SNPs corresponded to 194,313 variants and 537,957 effects. Most SNPs were downstream of genes $(30.29 \%)$, intergenic $(24.17 \%)$ or upstream of genes (31.37\%) (Fig. 2A).

\section{Genetic diversity and population structure}

According to the population structure evaluation, when $\mathrm{K}=2$, all 450 accessions were divided into two groups. Group 1 contained 396 accessions (88.0\%), mostly breeding varieties. Group 2 contained 54 (12.0\%) accessions, mostly cultivar introductions (Fig. 2B). When $\mathrm{K}=$ 3, the Group 1 subdivided into two groups, but Group 2 had no change. When $K=4$, the two groups subdivided from Group 1 maintained with a few individuals changed, but Group 2 subdivided into two groups. For K larger than 4, the two groups subdivided from Group 2 maintained, but the Group 1 subdivided into more groups (Supplementary Table 1).

To further illustrate the population structure of our research panel, principal component analysis (PCA) was performed. When the first and second eigenvectors were used, all accessions could be divided with four subgroups (Fig. 2C, Supplementary Fig. 1A). However, when divided into more than four groups, Group 2 was divided into more than two groups that showed indistinct boundaries (Fig. 2D, Supplementary Fig. 2B-F). Based on the above results, it is more representative when all 


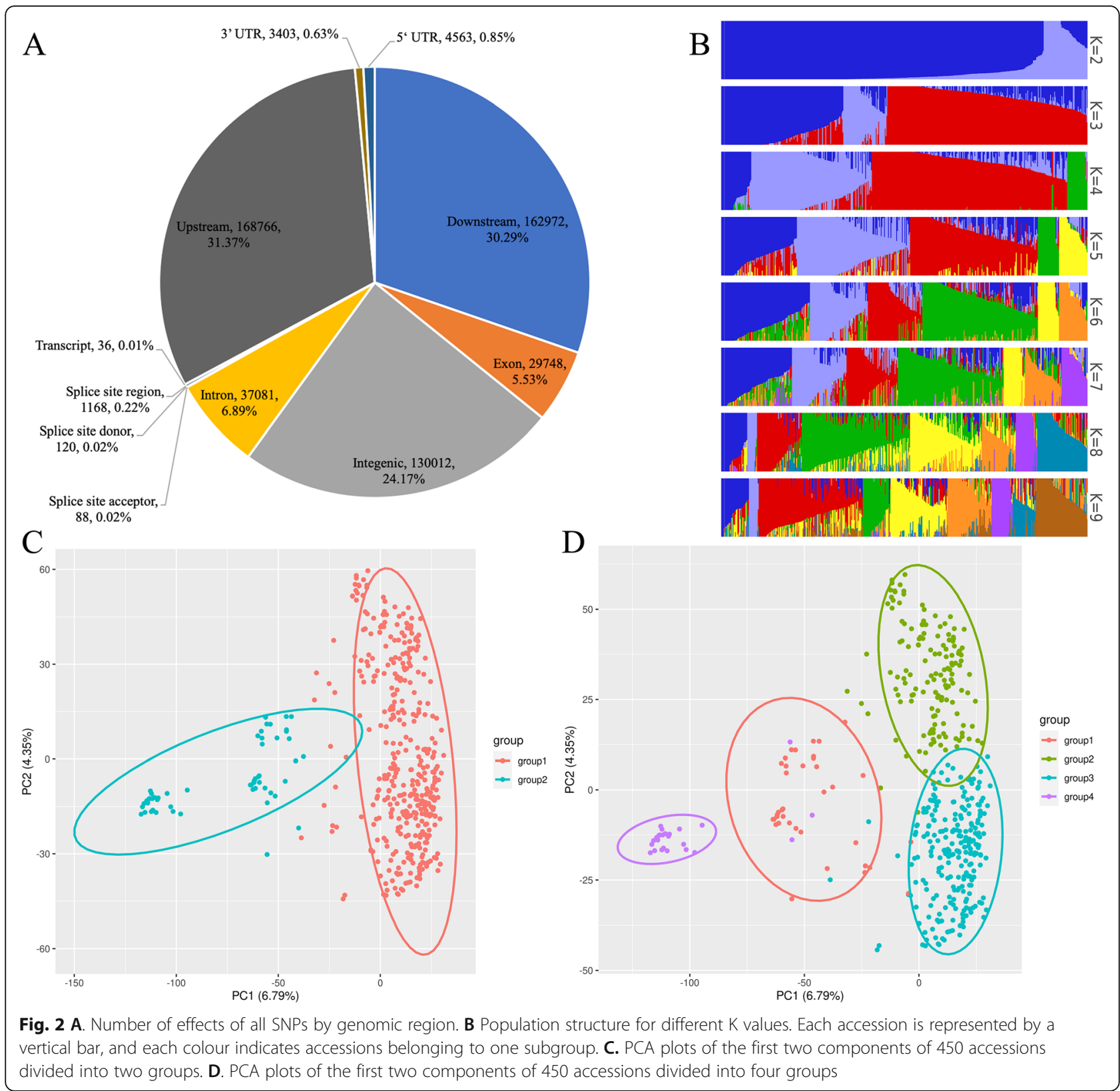

accessions were divided into four groups and suitable for further analysis. But greater difference were shown between Group 1 and Group 2 when $\mathrm{K}=2$.

A kinship matrix was calculated to detect the genetic relationship within the population. The coefficient of relatedness ranged from -0.25 to 2.01 , and a kinship heat map was drawn to visualize the relationships. It is clear that only the upper left corner has a relatively close relationship, and the other accessions have a lower coefficient of relatedness (Fig. 3), indicating that the population used in our research conform to a natural populations but with few relatedness between some accessions.

\section{Genome-wide association analysis}

The GLM found 597 SNPs to be significantly associated with four traits in total, but the MLM found only 322 (Table 1, Supplementary Table 3). For DH traits, no identical locus was found between the two models. By GLM, 144 loci were significantly associated with DH, and 25 loci were found at more than two locations on chromosomes 4, 6, 7 and 11 . However, by MLM, only 2 and 25 SNPs were found to be significantly associated with DH in Wuchang and Heihe (Table 1, Fig. 4A, Supplementary Fig. 5A). Twenty-five of them throughout all 12 chromosomes were from Heihe. Three SNPs on chromosome 7 shows larger effects and detected in 


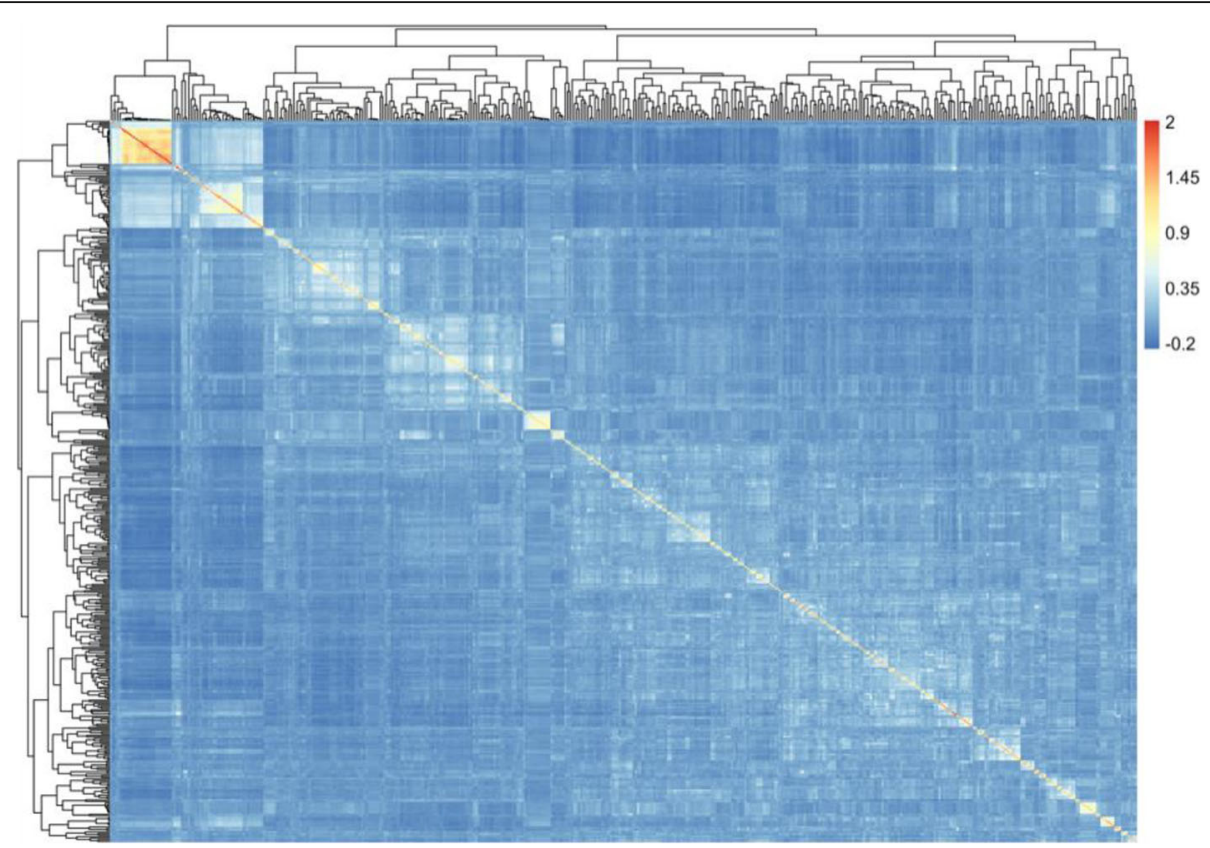

Fig. 3 Heatmap of genetic relationships. The colour change from cyan to red indicates an increase in the kinship value from -0.2 to 2

multi-environment (Table 2). For PH, 49 SNPs were found in three locations by GLM, 14, 6 and 29 SNPs in Harbin, Heihe and Wuchang, respectively, 2 of which on chromosome 11 and chromosome 12 were detected in both Heihe and Wuchang. Twenty-five SNPs were identified in only two locations by MLM, 1 and 24 in Heihe and Wuchang (Table 1, Fig. 4B, Supplementary Fig. 3B, Supplementary Fig. 5B). Twenty-three were detected by different methods at the same time or the same locations. Only 1 SNPs shows larger effects than other positions on chromosome 5 (Table 2). For TI, 53, 51, 9 and 3 SNPs were found by GLM in Harbin, Wuchang, Jiamusi and Heihe, respectively. By MLM, 41 and 2 were found in Wuchang and Harbin, but in Jiamusi and Heihe, only one for each location. Interestingly, all SNPs detected by MLM in Harbin, Jiamusi and Wuchang were also detected by GLM, but no SNPs were detected between any two locations by the same method (Table 1 , Fig. 4D, Supplementary Fig. 3-5D). Among these SNPs, 8 were larger effects and detected by two models. For $\mathrm{PW}$, the most significantly associated loci, 247 and 225,

Table 1 Summary of significantly associated loci identified by different methods

\begin{tabular}{|c|c|c|c|c|c|c|c|c|}
\hline & \multicolumn{4}{|c|}{ GLM } & \multicolumn{4}{|c|}{ MLM } \\
\hline & $\overline{\mathrm{HA}}$ & WU & $J A$ & $\overline{\mathrm{HE}}$ & HA & WU & $J A$ & $\mathrm{HE}$ \\
\hline$\overline{\mathrm{PH}}$ & 14 & 29 & - & 6 & - & 24 & - & 1 \\
\hline PW & 1 & 242 & - & 4 & 1 & 222 & - & 2 \\
\hline $\mathrm{TI}$ & 53 & 51 & 9 & 3 & 2 & 41 & 1 & 1 \\
\hline $\mathrm{DH}$ & 8 & 83 & 16 & 78 & - & 2 & - & 25 \\
\hline
\end{tabular}

were found by GLM and MLM, respectively. By GLM, only 1 and 4 SNPs were detected in Harbin and Heihe, respectively, but 242 were detected in Wuchang across all 12 chromosomes. Similar to GLM, MLM detected only 1 and 2 SNPs in Harbin and Heihe but 222 in Wuchang (Fig. 4C). Notably, all SNPs detected by MLM were also detected by GLM, except for 2 SNPs in Wuchang (Table 1, Supplementary Fig. 3-5C). Among these SNPs, 8 were larger effects and detected by two models. Interestingly, 3 SNPs (S4_32507995, S5_2003327, S11_ 8842451) were detected for both TI and PW by GLM (Table 2).

\section{Candidate gene identification}

A total of 317 SNPs detected either by two models or in more than two locations were selected for candidate gene identification. Of them, 223 SNPs were used for PW, and 19 missense variants, 1 splice region variant and 1 stop gained locus were identified (Table 3). Four missense variants were identified for TI, 3 for $\mathrm{PH}$ and only 1 for DH. Among these SNPs, Candidate genes of twenty two larger effect or pleiotropic SNPs were identified. Within gene regions, eleven SNPs were found. Two of them were 3 prime UTR variants, eight were found to be intron variants and only one SNP was synonymous variant. Eleven SNPs were located in intergenic region. The distance between SNPs and the nearest genes ranged from 0 to $18.9 \mathrm{~kb}$ (Table 4). All these genes execute unknown biology functions in $O$. sativa japonica group, according to the Rice Genome Annotation Project database. 

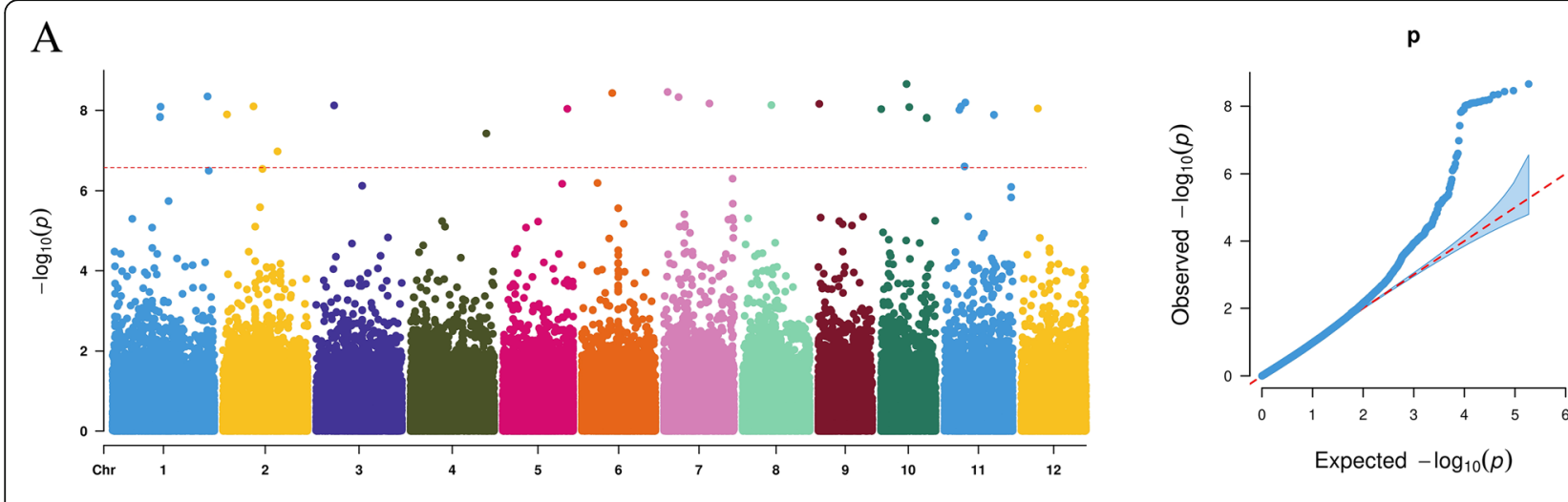

B
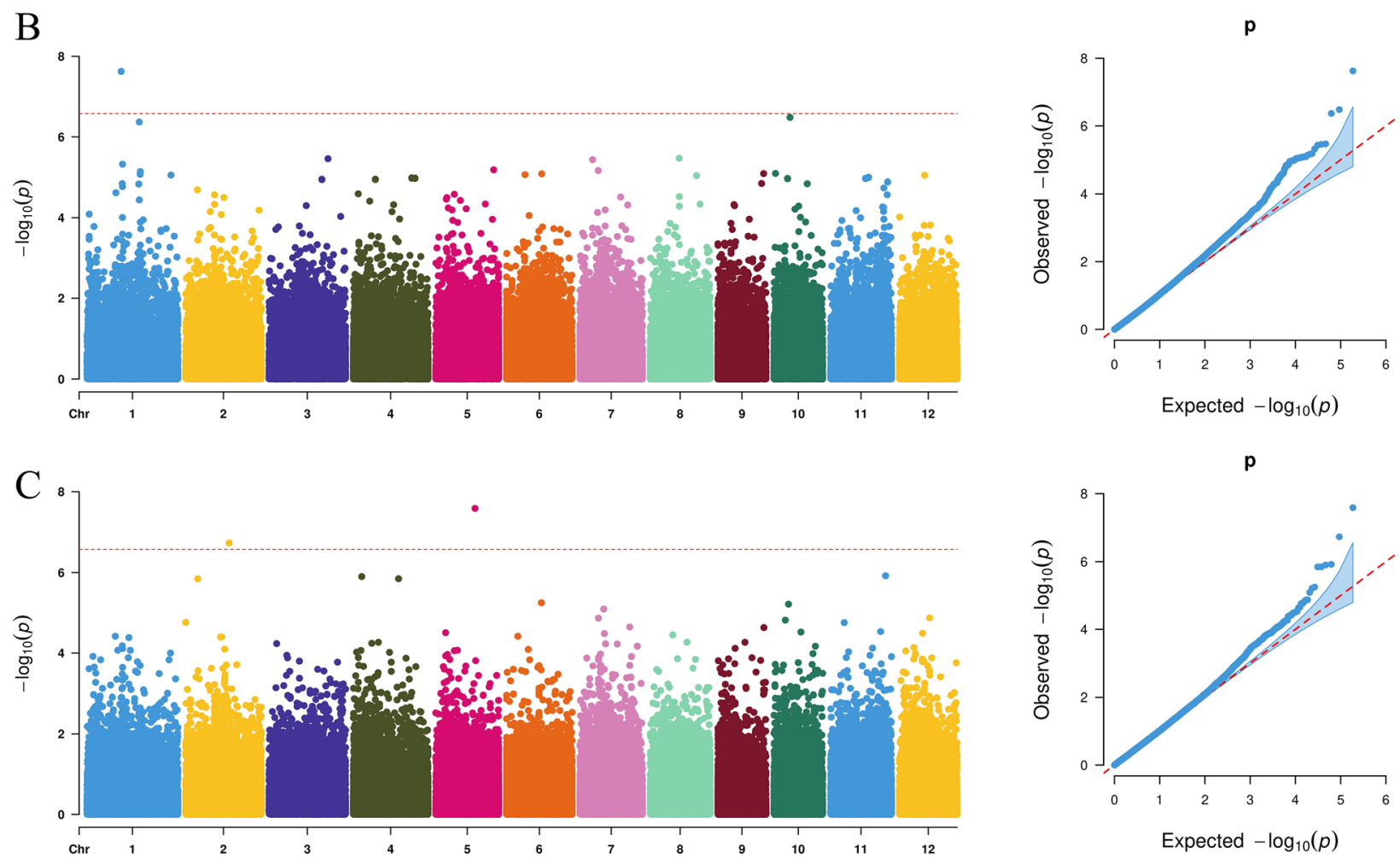

D
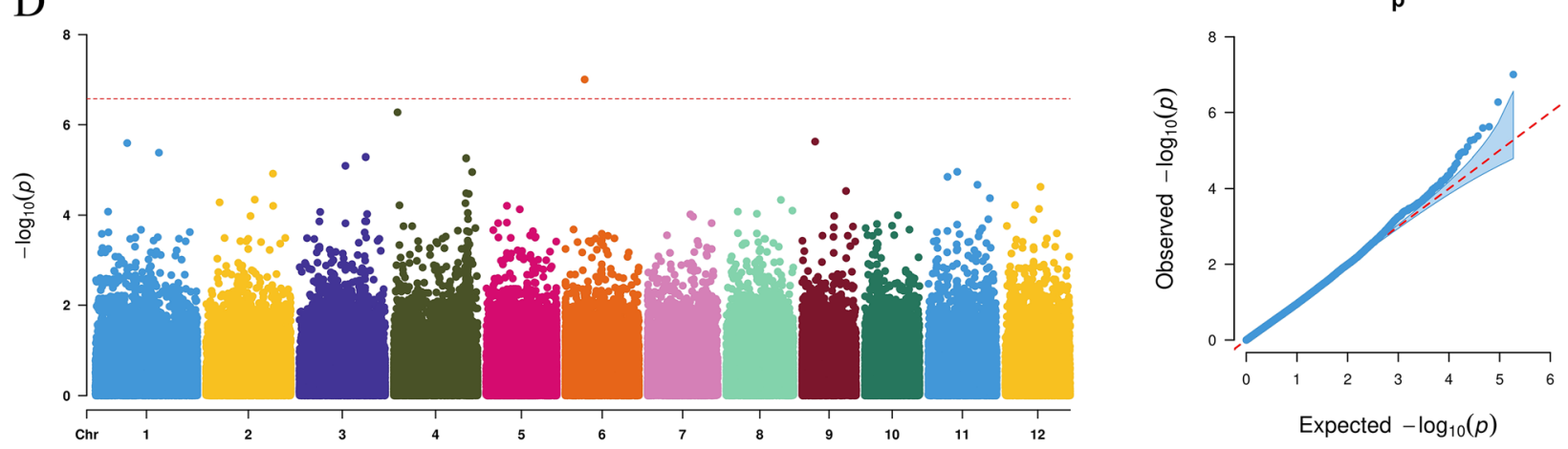

Fig. 4 Manhattan plots and QQ plots for the four traits in Heihe by MLM. A. Days to heading. B. Plant height. C. Panicle weight. D. Tiller number 
Table 2 Twenty larger effect SNPs and three pleiotropic SNPS

\begin{tabular}{|c|c|c|c|c|c|c|}
\hline Trait & Model & Location & Marker & $\mathrm{Chr}^{\mathrm{a}}$ & Position & $\operatorname{LOD}^{b}$ \\
\hline $\mathrm{DH}$ & GLM & $\mathrm{HA} / \mathrm{HE} / \mathrm{WU}$ & S7_8832791 & 7 & $8,832,791$ & $8.2062 / 13.9051 / 11.5954$ \\
\hline $\mathrm{DH}$ & GLM & $\mathrm{HA} / \mathrm{HE} / \mathrm{WU} / \mathrm{JA}$ & S7_29566846 & 7 & $29,566,846$ & 9.1926/14.0176/https://doi.org/10.8388/9.5634 \\
\hline $\mathrm{DH}$ & GLM & $\mathrm{HA} / \mathrm{HE} / \mathrm{WU} / \mathrm{JA}$ & S7_29238189 & 7 & $29,238,189$ & $8.7844 / 14.7984 / 11.7642 / 8.9488$ \\
\hline $\mathrm{PH}$ & GLM/MLM & WU & S5_11687290 & 5 & $11,687,290$ & $8.5651 / 8.1938$ \\
\hline $\mathrm{Tl}$ & GLM/MLM & WU & S2_9226198 & 2 & $9,226,198$ & $11.5279 / 10.4231$ \\
\hline $\mathrm{Tl}$ & GLM/MLM & WU & S2_13626408 & 2 & $13,626,408$ & $9.3376 / 8.1539$ \\
\hline $\mathrm{Tl}$ & GLM/MLM & WU & S2_34946356 & 2 & $34,946,356$ & $11.4379 / 10.0110$ \\
\hline $\mathrm{Tl}$ & GLM/MLM & WU & S4_7050530 & 4 & $7,050,530$ & $14.1317 / 11.9763$ \\
\hline $\mathrm{Tl}$ & GLM/MLM & WU & S7_3214984 & 7 & $3,214,984$ & https://doi.org/10.1969/8.6595 \\
\hline $\mathrm{Tl}$ & GLM/MLM & WU & S9_8477108 & 9 & $8,477,108$ & https://doi.org/10.5361/9.0133 \\
\hline $\mathrm{Tl}$ & GLM/MLM & WU & S11_8842451 & 11 & $8,842,451$ & $8.4670 / 8.1336$ \\
\hline $\mathrm{Tl}$ & GLM/MLM & WU & S11_26127237 & 11 & $26,127,237$ & $9.8739 / 8.1161$ \\
\hline PW & GLM/MLM & WU & S1_29521211 & 1 & $29,521,211$ & $17.7022 / 14.8049$ \\
\hline PW & GLM/MLM & WU & S3_13365688 & 3 & $13,365,688$ & https://doi.org/10.2207/9.2723 \\
\hline PW & GLM/MLM & WU & S4_16690715 & 4 & $16,690,715$ & https://doi.org/10.0706/9.1917 \\
\hline PW & GLM/MLM & WU & S4_22584137 & 4 & $22,584,137$ & https://doi.org/10.7148/8.4903 \\
\hline PW & GLM/MLM & WU & S6_8044409 & 6 & $8,044,409$ & https://doi.org/10.6151/8.7926 \\
\hline PW & GLM/MLM & WU & S7_9482292 & 7 & $9,482,292$ & $9.7741 / 8.7687$ \\
\hline PW & GLM/MLM & WU & S8_22791456 & 8 & $22,791,456$ & $16.3799 / 14.3599$ \\
\hline PW & GLM/MLM & WU & S11_5366552 & 11 & $5,366,552$ & https://doi.org/10.1661/8.6426 \\
\hline TI/PW & GLM & WU & S4_32507995 & 4 & $32,507,995$ & $9.0813 / 8.4376$ \\
\hline TI/PW & GLM & WU & S5_2003327 & 5 & $2,003,327$ & $7.2883 / 8.5209$ \\
\hline TI/PW & GLM & WU & S11_8842451 & 11 & $8,842,451$ & $8.4670 / 8.1495$ \\
\hline
\end{tabular}

${ }^{\mathrm{a}}$ Chromosome

LOD value in different locations or with different models

Table 3 Summary of annotation variants for four traits

\begin{tabular}{lllll}
\hline Traits & DH & PH & PW & TI \\
\hline number of SNPs & 25 & 25 & 223 & 44 \\
number of variants & 27 & 26 & 229 & 46 \\
number of effects & 81 & 64 & 589 & 133 \\
chromosome & $4,6,7,11$ & $1,3-8,10-12$ & $1-12$ & $1-12$ \\
3' UTR variant & - & - & 3 & - \\
5' UTR variant & 2 & - & 1 & - \\
downstream gene variant & 29 & 19 & 170 & 38 \\
frameshift variant & - & - & 1 & - \\
intergenic region & 16 & 18 & 143 & 31 \\
intron variant & 11 & 2 & 33 & 10 \\
missense variant & 1 & 3 & 19 & 4 \\
splice region variant & - & - & 1 & - \\
stop gained & - & - & 1 & - \\
synonymous variant & - & 3 & 31 & 4 \\
upstream gene variant & 22 & 19 & 187 & 46 \\
\hline
\end{tabular}

\section{Discussion}

Many models have been reported for use in GWAS, [23, 34, 35] and GLM and MLM are two that have been used frequently to analyse a variety of plants $[15,36]$. In GLM, the principal components or population structure needs to be taken into consideration as a fixed effect. However, in MLM, relative kinship should be added as a random effect, although the result is still less efficient for large data sets. Many other algorithms have been developed to address this problem, such as the compressed MLM, [35] efficient mixed-model association expedited (EMMAX) algorithm and [37] and factored spectrally transformed linear mixed model (FaST-LMM) [38]. When different models are compared, some of them show high statistical power but low computational speed, while others show intermediate statistical power but very fast computational speed [26]. For further gene screening, these models should be adapted to increase the accuracy of the associations and narrow down the possible associated interval.

Association analysis was first used on populations of unrelated human individuals, [39] but it is difficult to 
Table 4 Candidate genes identified from 22 detected SNPs for 4 traits

\begin{tabular}{|c|c|c|c|c|}
\hline SNP & $\mathrm{Chr}^{\mathrm{a}}$ & Gene ID ${ }^{b}$ & Annotation & Distance $(\mathbf{k b})^{c}$ \\
\hline S1_29521211 & 1 & LOC_Os01g51330 & 3 prime UTR variant & 0.0 \\
\hline S2_9226198 & 2 & LOC_Os02g16230 & intergenic region & 2.3 \\
\hline S2_13626408 & 2 & LOC_Os02g22820 & intergenic region & 18.9 \\
\hline S2_34946356 & 2 & LOC_Os02g57080 & intron variant & 0.0 \\
\hline S3_13365688 & 3 & LOC_Os03g23110 & intron variant & 0.0 \\
\hline S4_7050530 & 4 & LOC_Os04g12744 & intergenic region & 2.5 \\
\hline S4_16690715 & 4 & LOC_Os04g28234 & 3 prime UTR variant & 0.0 \\
\hline S4_22584137 & 4 & LOC_Os04g37960 & intron variant & 0.0 \\
\hline S4_32507995 & 4 & LOC_Os04g54690 & intergenic region & $0.0^{d}$ \\
\hline S5_11687290 & 5 & LOC_Os05g19990 & synonymous variant & 0.0 \\
\hline S5_2003327 & 5 & LOC_Os05g04370 & intergenic region & 0.5 \\
\hline S6_8044409 & 6 & LOC_Os06g14406 & intron variant & 0.0 \\
\hline S7_8832791 & 7 & LOC_Os07g15330 & intron variant & 0.0 \\
\hline S7_29566846 & 7 & LOC_Os07g49370 & intron variant & 0.0 \\
\hline S7_29238189 & 7 & LOC_Os07g48870 & intergenic region & 3.9 \\
\hline S7_3214984 & 7 & LOC_Os07g06610 & intergenic region & 0.9 \\
\hline S7_9482292 & 7 & LOC_Os07g16240 & intergenic region & 3.4 \\
\hline S8_22791456 & 8 & LOC_Os08g36160 & intergenic region & 2.5 \\
\hline S9_8477108 & 9 & LOC_Os09g14350 & intron variant & 0.0 \\
\hline S11_8842451 & 11 & LOC_Os11g15590 & intron variant & 0.0 \\
\hline S11_26127237 & 11 & LOC_Os11g43300 & intergenic region & 3.3 \\
\hline S11_5366552 & 11 & LOC_Os11g10010 & intergenic region & 0.9 \\
\hline
\end{tabular}

${ }^{\mathrm{a} C h r o m o s o m e}$

${ }^{\mathrm{b}}$ Gene ID from MSU genome annotation database (version 7.0)

${ }^{\mathrm{C}}$ The distance between SNPs and the nearest gene. The $0 \mathrm{~kb}$ means that the SNP located inside the gene

${ }^{\mathrm{d}}$ The distance between the SNP S4_32507995 and the gene LOC_Os04g54690 is 28 bp

collect natural plant populations with distant genetic relationships in a local area. Although many accessions collected in this research were from South Korea, Russia and Japan, many of them were elite varieties derived from the same ancestral parents. Meanwhile, information on some cultivars was lost, resulting in unknown origins. Genetic population analysis also showed that the distinctions among some of these accessions were obscure (Fig. 3), so further research is needed to optimize the population structure and screen the research panels to obtain a clearer population structure $[40,41]$.

Principal component analysis has been shown to be a substitute for population structure in GWAS [42]. Therefore, we chose the first five components as the population structure matrix to conduct a GWAS. The eigenvalue derived from PCA was proportionally low because of the large population scale $(6.79 \%$ for the first principal component, data not shown). The smooth downward trend of the eigenvalue made it difficult to choose the number of components for association analysis (Supplementary Fig. 6 ) [43]. The different ways of dividing population groups by population structure and PCA eigenvector also made it difficult to select a population structure matrix. Therefore, more population structure matrices may be needed for further analysis to locate the key associated SNPs.

A total of $144 \mathrm{DH}$-related SNPs were detected in four locations by GLM, and 25 of them were detected in more than two locations, which implied that even at different latitudes, heading date was functionally affected by the same genes. Little attention has been given to the associations of PW in cereal crops, but relationships with grain yield and rice quality have been reported [44, 45]. The PW is also used as a main trait for association analysis in rice [46]. However, in our study, too many loci were associated with PW across all 12 chromosomes (Fig. 4C, Supplementary Fig. 3-5C). Therefore, it was not easy to identify the true related genes among these SNPs. More association analysis models may be needed to narrow down the candidate genes. In addition, PW is a comprehensive trait consisting of many factors, such as panicle length, number of grains, and grain weight, 
which adds to the difficulties of detecting associated sites. A separate analysis of this trait may be needed for further association studies.

From the Manhattan plots, it is obvious that DH shows significant peak values by GLM: 2 peak values on chromosome 2, 1 on chromosome 4 and 2 on chromosome 7 (Supplementary Fig. 7). The peak value of chromosome 4 was located at $14,818,439 \mathrm{bp}$ in the $5^{\prime}$ UTR of the LOC_Os04g25560 gene, which is referred to as the OsSCP23 (putative serine carboxypeptidase homologue) gene. The two peak values of chromosome 7 located at 8,832,791 bp and 29,566,846 bp corresponded to the intron variant LOC_Os07g15330 and the intron variant LOC_Os07g49370, respectively. Both putative genes have unclear functions. Notably, these two genes were located on chromosome 7, close to two reported heading date genes, Ghd7 (LOC_Os07g15770) and DTH7 (LOC_Os0749460), which are approximately $320 \mathrm{~kb}$ and $50 \mathrm{~kb}$ in length, respectively [47, 48]. In chromosome 2, the location of the peak value is far from the reported genes LOC_Os02g39710 and LOC_ Os02g49230 $[49,50]$. It may be concluded that many factors can cause false positive results in GWAS, so a wider screening range is needed to choose the affected genes around the associated SNPs. LD blocks will also provide a reference criterion for the range. Various issues need to be considered for further gene screening.

\section{Conclusion}

In this study, 450 accessions were used to perform whole-genome resequencing, and 189,019 SNPs were used for GWAS after filtering according to the MAF, missing genotype rate and $r^{2}$ value. Bonferroni correction was used to set the threshold of significantly associated SNPs to $-\log _{10}(\mathrm{P}) \geq 6.58$. In total, 597 and 322 significantly associated loci were detected for the four traits by GLM and MLM, respectively. After filtered, 22 larger effect or pleiotropic SNPs for the four traits were used to identify candidate genes. Eleven SNPs were identified within coding regions, two of them were located in 3' UTR, eight in intron region and one in coding region. The rest of 11 SNPs were found to be located in intergenic region.

All these candidate genes associated with the four yield traits could be used for further gene identification or fine mapping, and related SNPs will also provide guidance for rice breeding in high-latitude areas.

\section{Materials and methods Plant materials}

A collection of 450 temperate japonica rice varieties was used as a GWAS panel, including landraces and cultivars collected from Japan, North Korea, Russia, Heilongjiang Province, Jilin Province and Liaoning Province in China, and other unknown origins (Supplementary Table 1). Many landraces and foreign varieties have been introduced into Heilongjiang Province of China in recent decades. Moreover, a number of intermediate varieties were added to the panel for further analyses.

\section{Field cultivation and management}

All materials were planted in the field in 2015. Four experimental fields were located at Wuchang $\left(44.9^{\circ} \mathrm{N}\right.$, $\left.127.2^{\circ} \mathrm{E}\right)$, Harbin $\left(45.8^{\circ} \mathrm{N}, 126.6^{\circ} \mathrm{E}\right)$, Jiamusi $\left(46.8^{\circ} \mathrm{N}\right.$, $\left.130.4^{\circ} \mathrm{E}\right)$ and Heihe $\left(50.2^{\circ} \mathrm{N}, 127.5^{\circ} \mathrm{E}\right)$ in Heilongjiang Province, and all these locations were used as paddy fields for successive years. Wuchang is the most fertile black land of China, with approximately 145 days above the minimal temperature. At higher latitudes, Harbin and Jiamusi have fewer days above the minimal temperature. Heihe is not only the highest latitude of the Chinese temperate zone but also the highest latitude of the world where rice is cultivated. The growth season in Heihe is less than 120 days. Experiments were constructed with a complete randomized design. Ten plants of each accession were used for a single row with $13 \mathrm{~cm}$ spacing within the plants and $30 \mathrm{~cm}$ spacing between the rows. Field management was conducted normally for a local paddy field.

\section{Phenotypic evaluations and data statistics}

The PH was evaluated before harvest and was measured from ground to the highest panicle tip. The TI was counted in each plant with panicles. The main panicle in each plant was collected and weighed from the rachis internode to obtain the PW. The mean of three plants was calculated as the final value. The heading date was noted when over $50 \%$ of plants in a row were heading, and the number of days from sowing to heading was used as DH for further analysis. Phenotype statistics and distribution analyses were performed with the R/base package. Analyses of variance for the four traits were performed using the lmerTest package in R by Student's $t$ test with a confidence level of $\alpha<0.001$ (https://cran.rproject.org/web/packages/lmerTest/index.html). Lines and locations were treated as random effects, and traits were treated as fixed effects with the formula Trait (1|line) + (1|location) by lme4/R (https://github.com/ lme4/lme4). The broad-sense heritability $\left(H^{2}\right)$ of the four traits was calculated by the following equation: $\mathrm{H}^{2}=\mathrm{Vg} /$ $(\mathrm{Vg}+\mathrm{Ve} / \mathrm{L})$, where $\mathrm{Vg}$ is the variance of genotypes, $\mathrm{Ve}$ is the variance of environments, and $\mathrm{L}$ is the number of locations. Statistical plots were drawn with ggplot2/R (http://had.co.nz/ggplot2/).

\section{Genome resequencing and genotype filtering}

Young leaves were collected from each accession, and genomic deoxyribonucleic acid (DNA) was isolated by a 
rapid method to obtain high-quality total DNA (DOI: https://doi.org/10.21769/BioProtoc.1010106). Paired-end libraries were constructed and sequenced on an Illumina HiSeq sequencing system (Illumina, USA). The Nipponbare genome (MSU version 7.0) was used as a reference genome. All reads were aligned to the reference genome with the Burrows-Wheeler Alignment (BWA) tool [51]. After the alignment, quality control was performed with SAMtools (Ver. 1.7), [52] and the Genome Analysis Toolkit was used for SNP calling (GATK, v3.446). The UnifieldGenotyper of GATK was used for multiple SNP calling [53]. Genotype imputation was performed using Tassel (Version 5.0) with the LD KNNi Imputation plugin [27].

\section{Minor allele frequency and linkage disequilibrium}

The MAF was calculated with Plink (Version 1.9, http:// www.cog-genomics.org/plink2/). After genotype imputation, SNPs were filtered by Plink with thresholds of MAF greater than 0.05 and a missing genotype rate greater than 0.2 . Whole-genome LD decay was estimated by pairwise squared correlation coefficients $\left(r^{2}\right)$ between SNPs in PopLDdecay [54]. The pairwise $r^{2}$ value was calculated when all accessions were divided into four groups by population genetic analysis. The LD decay distances were determined where the average $r^{2}$ dropped to half of its maximum value. The SNPs were further filtered according to the $r^{2}$ value in Plink with parameters --indep-pairwise 50100.2 .

\section{Population genetic analyses}

Population structure analysis, including group estimation, best $\mathrm{K}$ value selection and population structure plotting, was performed with fastSTRUCTURE (version 1.0) by the structure, chooseK and distruct plugins, respectively [55]. The PCA and kinship matrix were calculated within TASSEL (Version 5.0) [27]. The first five components were used for further association analysis. The PCA plots of the first two components were drawn with different groups predicted from $\mathrm{K}$ values. The kinship heatmap was drawn with pheatmap/R (Version 1.0.12, https://cran.r-project.org/web/packages/ pheatmap/index.html). The clustering distance of rows and columns used correlation as its parameter, and the complete parameter was used for the clustering method.

\section{Genome-wide association analysis}

The GWAS analysis was carried out with TASSEL 5, and GLM and MLM were used to detect significantly associated loci. After filtering by LD value, 189,019 SNPs were used for association analysis with a threshold LOD value of 6.58. Genotypes and phenotypes were used in the GLM model with the first five components of PCA as the population structure matrix. However, in the
MLM, a kinship matrix was also added as a relatedness covariation. The threshold values for associated SNPs were obtained by the Bonferroni correction, which was calculated as follows: $-\log _{10}(\mathrm{P}) \geq-\log _{10}(0.05 / 189,019) \approx$ 6.58. Manhattan plots and QQ plots were drawn by CMplot/R (Version 3.6.2, https://cran.r-project.org/web/ packages/CMplot/).

\section{Candidate gene identification}

Loci that were detected in more than two locations or by both methods were used for candidate gene identification. The Nipponbare genome (MSU version 7.0, http://rice.plantbiology.msu.edu/) annotation database was used as a reference database. SnpEff (Version $4.3 \mathrm{~T}$ ) was used to annotate significantly associated SNPs [56].

\section{Abbreviations}

GWAS: Genome-wide association studies; GLM: General linear model; MLM: Mixed linear model; DH: Days to heading; PH: Plant height; PW: Panicle weight; TI: Tiller number; SNP: Single-nucleotide polymorphism;

DNA: Deoxyribonucleic acid; QTL: Quantitative trait locus; MSU: Michigan State University; MAF: Minor allele frequency; BWA: Burrows-Wheeler Alignment; LD: Linkage disequilibrium; PCA: Principal component analysis; LOD: Likelihood of odds; UTR: Untranslated region

\section{Supplementary Information}

The online version contains supplementary material available at https://doi. org/10.1186/s12863-021-00995-y.

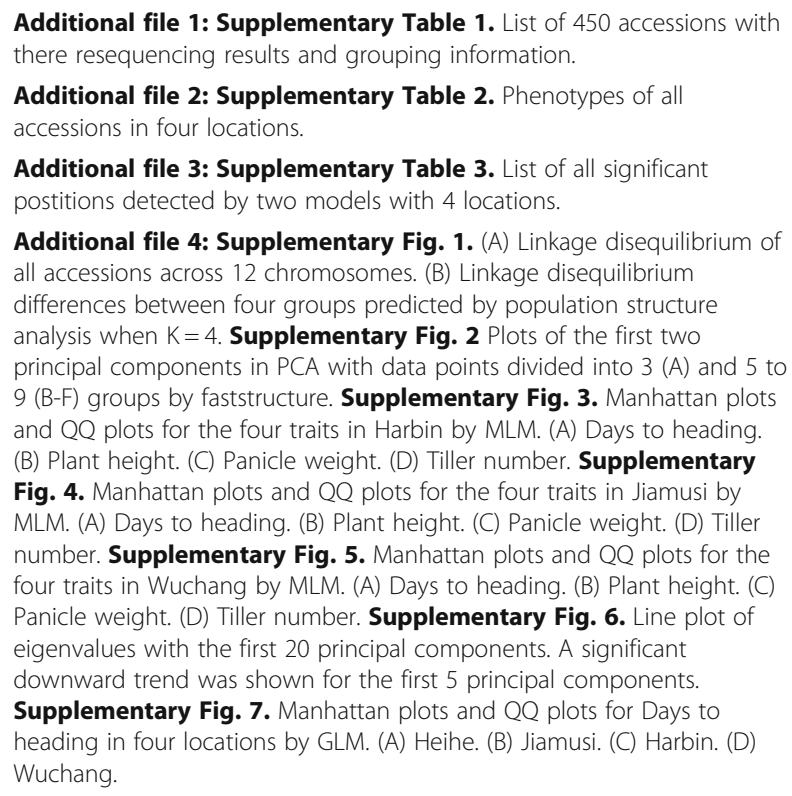

Additional file 2: Supplementary Table 2. Phenotypes of all accessions in four locations.

Additional file 3: Supplementary Table 3. List of all significant postitions detected by two models with 4 locations.

Additional file 4: Supplementary Fig. 1. (A) Linkage disequilibrium of all accessions across 12 chromosomes. (B) Linkage disequilibrium differences between four groups predicted by population structure analysis when $K=4$. Supplementary Fig. 2 Plots of the first two principal components in PCA with data points divided into $3(A)$ and 5 to 9 (B-F) groups by faststructure. Supplementary Fig. 3. Manhattan plots and QQ plots for the four traits in Harbin by MLM. (A) Days to heading. (B) Plant height. (C) Panicle weight. (D) Tiller number. Supplementary Fig. 4. Manhattan plots and QQ plots for the four traits in Jiamusi by MLM. (A) Days to heading. (B) Plant height. (C) Panicle weight. (D) Tiller number. Supplementary Fig. 5. Manhattan plots and QQ plots for the four traits in Wuchang by MLM. (A) Days to heading. (B) Plant height. (C) Panicle weight. (D) Tiller number. Supplementary Fig. 6. Line plot of eigenvalues with the first 20 principal components. A significant downward trend was shown for the first 5 principal components. Supplementary Fig. 7. Manhattan plots and QQ plots for Days to heading in four locations by GLM. (A) Heihe. (B) Jiamusi. (C) Harbin. (D) Wuchang.

\section{Acknowledgements}

We thank Dr. Chengzhi Liang at the Institute of Genetics and Developmental Biology, Chinese Academy of Sciences, for resequencing all accessions and performing primary raw data filtering and integration.

\section{Authors' contributions}

Guomin Zhang and Ying Wang conceived and designed the experiment. Rongsheng Wang performed data analysis and wrote the manuscript. Hongru Gao, Lingwei Deng, Nanbo Wang, Kun Li, Wei Zhang and Yongli Wang conducted phenotype surveys and trait measurements. Juntao Ma, 
Jun Zhang, Fengchen Mu and Hui Liu contributed to field management and coordination. The author(s) read and approved the final manuscript.

\section{Funding}

This work was partially supported by the Chinese Academy of Sciences "Strategic Priority Program" subproject fund (XDA24030101-4) and Heilongjiang Academy of Agricultural Sciences Academy-level Research Project funding (2020YYYF059).

\section{Availability of data and materials}

Raw reads of 450 accessions used in this study were a part of BioProject PRJCA000322 in the National Genomics Data Center (https://ngdc.cncb.ac.cn) and can be accessed by accession ID listed in Supplementary Table 1.

\section{Declarations}

Ethics approval and consent to participate

Not applicable.

\section{Consent for publication}

Not applicable.

\section{Competing interests}

The authors declare that they have no competing interests.

\section{Received: 30 March 2021 Accepted: 13 September 2021}

\section{Published online: 05 October 202}

\section{References}

1. Wing RA, Purugganan MD, Zhang Q. The rice genome revolution: from an ancient grain to green super Rice. Nat Rev Genet. 2018;19(8):505-17. https:// doi.org/10.1038/s41576-018-0024-Z.

2. Wang W, Mauleon R, Hu Z, Chebotarov D, Tai S, Wu Z, et al. Genomic variation in 3,010 diverse accessions of Asian cultivated rice. Nature. 2018; 557(7703):43-9. https://doi.org/10.1038/s41586-018-0063-9.

3. Hu Z, Wang W, Wu Z, Sun C, Li M, Lu J, et al. Novel sequences, structural variations and gene presence variations of Asian cultivated rice. Sci Data. 2018:5(1):180079. https://doi.org/10.1038/sdata.2018.79.

4. Choi JY, Platts AE, Fuller DQ, Hsing Y-I, Wing RA, Purugganan MD. The Rice paradox: multiple origins but single domestication in Asian Rice. Mol Biol Evol. 2017;34(4):969-79. https://doi.org/10.1093/molbev/msx049.

5. Caicedo AL, Williamson SH, Hernandez RD, Boyko A, Fledel-Alon A, York TL, et al. Genome-wide patterns of nucleotide polymorphism in domesticated rice. PLoS Genet. 2007:3(9):1745-56. https://doi.org/10.13 71/journal.pgen.0030163

6. Civán $\mathrm{P}, \mathrm{Craig} H, \mathrm{Cox} C \mathrm{C}$, Brown TA. Three geographically separate domestications of Asian rice. Nat Plants. 2015;1(11):15164. https://doi.org/1 0.1038/nplants.2015.164.

7. Olsen KM, Caicedo AL, Polato N, McClung A, McCouch S, Purugganan MD Selection under domestication: evidence for a sweep in the rice waxy genomic region. Genetics. 2006;173(2):975-83. https://doi.org/10.1534/ genetics.106.056473.

8. Olsen KM, Purugganan MD. Molecular evidence on the origin and evolution of glutinous rice. Genetics. 2002;162(2):941-50. https://doi.org/10.1093/ genetics/162.2.941

9. Zhang Z, Li J, Pan Y, Li J, Zhou L, Shi H, et al. Natural variation in CTB4a enhances rice adaptation to cold habitats. Nat Commun. 2017:8:14788.

10. Ashikari M, Matsuoka M. QTL detection and its application to rice breeding. Plant Cell Physiol. 2006:47:S14.

11. Huang XQ, Coster H, Ganal MW, Roder MS. Advanced backcross QTL analysis for the identification of quantitative trait loci alleles from wild relatives of wheat ( Triticum aestivum L.). Theor Appl Genet. 2003;106: 1379-89.

12. Miura K, Ashikari M, Matsuoka M. The role of QTLs in the breeding of highyielding rice. Trends Plant Sci. 2011;16(6):319-26. https://doi.org/10.1016/j. tplants.2011.02.009.

13. $Y u$ J, Buckler ES. Genetic association mapping and genome organization of maize. Curr Opin Biotech. 2006;17(2):155-60. https://doi.org/10.1016/j. copbio.2006.02.003.

14. Huang X, Zhao Y, Wei X, Li C, Wang A, Zhao Q, et al. Genome-wide association study of flowering time and grain yield traits in a worldwide collection of rice germplasm. Nat Genet. 2012;44(1):32-9. https://doi.org/1 $0.1038 /$ ng.1018

15. Huang X, Wei X, Sang T, Zhao Q, Feng Q, Zhao Y, et al. Genome-wide association studies of 14 agronomic traits in rice landraces. Nat Genet. 2010; 42(11):961-7. https://doi.org/10.1038/ng.695.

16. Huang X, Yang S, Gong J, Zhao Q, Feng Q, Zhan Q, et al. Genomic architecture of heterosis for yield traits in rice. Nature. 2016;537(7622):62933. https://doi.org/10.1038/nature19760.

17. Tian F, Bradbury PJ, Brown PJ, Hung H, Sun Q, Flint-Garcia S, et al. Genomewide association study of leaf architecture in the maize nested association mapping population. Nat Genet. 2011;43(2):159-62. https://doi.org/10.1038/ ng.746.

18. Dell'Acqua M, Gatti DM, Pea G, Cattonaro F, Coppens F, Magris G, et al. Genetic properties of the MAGIC maize population: a new platform for high definition QTL mapping in Zea mays. Genome Biol. 2015;16(1):167. https:// doi.org/10.1186/s13059-015-0716-z.

19. Gan X, Stegle O, Behr J, Steffen JG, Drewe P, Hildebrand KL, et al. Multiple reference genomes and transcriptomes for Arabidopsis thaliana. Nature. 2011;477(7365):419-23. https://doi.org/10.1038/na ture 10414.

20. Pan Q, Xu Y, Li K, Peng Y, Zhan W, Li W, et al. The genetic basis of plant architecture in 10 maize recombinant inbred line populations. Plant Physiol. 2017;175(2):858-73. https://doi.org/10.1104/pp.17.00709.

21. Pan Q, Li L, Yang X, Tong H, Xu S, Li Z, et al. Genome-wide recombination dynamics are associated with phenotypic variation in maize. New Phytol. 2016;210(3):1083-94. https://doi.org/10.1111/nph.13810.

22. Liu X, Huang M, Fan B, Buckler ES, Zhang Z. Iterative usage of fixed and random effect models for powerful and efficient genome-wide association studies. PLoS Genet. 2016;12(2):e1005767. https://doi.org/10.1371/journal. pgen.1005767.

23. Wang S-B, Feng J-Y, Ren W-L, Huang B, Zhou L, Wen Y-J, et al. Improving power and accuracy of genome-wide association studies via a multi-locus mixed linear model methodology. Sci Rep-UK. 2016;6(1):19444. https://doi. org/10.1038/srep19444.

24. Larsson SJ, Lipka AE, Buckler ES. Lessons from Dwarf8 on the strengths and weaknesses of structured association mapping. PLoS Genet. 2013:9(2): e1003246. https://doi.org/10.1371/journal.pgen.1003246.

25. Schraiber JG, Akey JM. Methods and models for unravelling human evolutionary history. Nat Rev Genet. 2015;16(12):727-40. https://doi.org/10.1 038/nrg4005.

26. Xiao Y, Liu H, Wu L, Warburton M, Yan J. Genome-wide association studies in maize: praise and stargaze. Mol Plant. 2017;10(3):359-74. https://doi.org/1 0.1016/j.molp.2016.12.008

27. Bradbury PJ, Zhang Z, Kroon DE, Casstevens TM, Ramdoss Y, Buckler ES TASSEL: software for association mapping of complex traits in diverse samples. Bioinformatics. 2007;23(19):2633-5. https://doi.org/10.1093/ bioinformatics/btm308.

28. Francis RM. Pophelper: an R package and web app to analyse and visualize population structure. Mol Ecol Resour. 2017;17(1):27-32. https://doi.org/1 0.1111/1755-0998.12509

29. Chen W, Gao Y, Xie W, Gong L, Lu K, Wang W, et al. Genome-wide association analyses provide genetic and biochemical insights into natural variation in rice metabolism. Nat Genet. 2014;46(7):714-21. https://doi.org/1 0.1038/ng.3007

30. Si L, Chen J, Huang X, Gong H, Luo J, Hou Q, et al. OsSPL13 controls grain size in cultivated rice. Nat Genet. 2016;48(4):447-56. https://doi.org/10.1038/ ng. 3518.

31. Xing Y, Zhang Q. Genetic and molecular bases of rice yield. Annu Rev Plant Biol. 2010;61(1):421-42. https://doi.org/10.1146/annurev-arplant-042 809-112209.

32. Yano M, Sasaki T. Genetic and molecular dissection of quantitative traits in rice. Plant Mol Biol. 1997;35(1/2):145-53. https://doi.org/10.1023/A:10057642 09331.

33. Xu X, Liu X, Ge S, Jensen JD, Hu F, Li X, et al. Resequencing 50 accessions of cultivated and wild rice yields markers for identifying agronomically important genes. Nat Biotechnol. 2012;30(1):105-11. https://doi.org/10.1038/ nbt.2050

34. Yu J, Pressoir G, Briggs WH, Vroh Bi I, Yamasaki M, Doebley JF, et al. A unified mixed-model method for association mapping that accounts for multiple levels of relatedness. Nat Genet. 2006;38(2):203-8. https://doi.org/1 $0.1038 /$ ng 1702 . 
35. Zhang Z, Ersoz E, Lai C-Q, Todhunter RJ, Tiwari HK, Gore MA, et al. Mixed linear model approach adapted for genome-wide association studies. Nat Genet. 2010;42(4):355-60. https://doi.org/10.1038/ng.546.

36. Wu C, Mozzoni LA, Moseley D, Hummer W, Ye H, Chen P, et al. Genomewide association mapping of flooding tolerance in soybean. Mol Breeding 2019:40(1):4. https://doi.org/10.1007/s11032-019-1086-0.

37. Kang HM, Sul JH, Service SK, Zaitlen NA, Kong S, Freimer NB, et al. Variance component model to account for sample structure in genome-wide association studies. Nat Genet. 2010;42(4):348-54. https:// doi.org/10.1038/ng.548.

38. Lippert C, Listgarten J, Liu Y, Kadie CM, Davidson Rl, Heckerman D. FaST linear mixed models for genome-wide association studies. Nat Methods. 2011;8(10):833-5. https://doi.org/10.1038/nmeth.1681.

39. Risch N, Merikangas K. The future of genetic studies of complex human diseases. Science. 1996;273(5281):1516-7. https://doi.org/10.1126/science.2 73.5281.1516.

40. Kang HM, Zaitlen NA, Wade CM, Kirby A, Heckerman D, Daly MJ, et al. Efficient control of population structure in model organism association mapping. Genetics. 2008;178(3):1709-23. https://doi.org/10.1534/genetics.1 07.080101

41. Martin LS, Eskin E. Review: population structure in genetic studies: confounding factors and mixed models. bioRxiv. 2017; https://doi.org/10.11 01/092106.

42. Zhao H, Nandita M, KP A, NK L, Rebbeck Timothy R. A practical approach to adjusting for population stratification in genome-wide association studies: principal components and propensity scores (PCAPS). Stat Appl Genet Mol. 2018;17:1-12.

43. Nick P, Alkes L, Price $D$, et al. Population structure and eigenanalysis. PLoS Genet. 2006;2(12):e190.

44. Bian J, Ren G, Han C, Xu F, Qiu S, Tang J, et al. Comparative analysis on grain quality and yield of different panicle weight indica-japonica hybrid rice (Oryza sativa L.) cultivars. J Integr Agr. 2020;19(4):999-1009. https://doi. org/10.1016/S2095-3119(19)62798-X.

45. Chapko LB, Brinkman MA. Interrelationships between panicle weight, grain yield, and grain yield components in oat. Crop Sci. 1991;31(4):878-82. https://doi.org/10.2135/cropsci1991.0011183X003100040007x.

46. Crowell S, Korniliev P, Falcão A, Ismail A, Gregorio G, Mezey J, et al. Genome-wide association and high-resolution phenotyping link Oryza sativa panicle traits to numerous trait-specific QTL clusters. Nat Commun. 2016;7(1):10527. https://doi.org/10.1038/ncomms10527.

47. Koo BH, Yoo SC, Park JW, Kwon CT, Lee BD, An G, et al. Natural variation in OsPRR37 regulates heading date and contributes to rice cultivation at a wide range of latitudes. Mol Plant. 2013;6(6):1877-88. https://doi.org/10.1 093/mp/sst088.

48. Xue $W$, Xing $Y$, Weng $X$, Zhao $Y$, Tang $W$, Wang $L$, et al. Natural variation in Ghd7 is an important regulator of heading date and yield potential in rice. Nat Genet. 2008;40(6):761-7. https://doi.org/10.1038/ng.143.

49. Lee YS, Jeong DH, Lee DY, Yi J, Ryu CH, Kim SL, et al. OsCOL4 is a constitutive flowering repressor upstream of Ehd1 and downstream of OsphyB. Plant J. 2010;63(1):18-30. https://doi.org/10.1111/j.1365-313X.2010.04226.x

50. Li J, Xu R, Wang CC, Qi L, Zheng XM, Wang WS, et al. A heading date QTL, qHD7.2, from wild rice (Oryza rufipogon) delays flowering and shortens panicle length under long-day conditions. Sci Rep-UK. 2018;8:2928. https:// doi.org/10.1038/s41598-018-21330-z.

51. Li H, Durbin R. Fast and accurate short read alignment with burrowswheeler transform. Bioinformatics. 2009;25(14):1754-60. https://doi.org/10.1 093/bioinformatics/btp324.

52. Li H. A statistical framework for SNP calling, mutation discovery, association mapping and population genetical parameter estimation from sequencing data. Bioinformatics. 2011;27(21):2987-93. https://doi.org/10.1093/bioinforma tics/btr509.

53. McKenna A, Hanna M, Banks E, Sivachenko A, Cibulskis K, Kernytsky A, et al. The genome analysis toolkit: a MapReduce framework for analyzing nextgeneration DNA sequencing data. Genome Res. 2010;20(9):1297-303. https://doi.org/10.1101/gr.107524.110.

54. Zhang C, Dong S-S, Xu J-Y, He W-M, Yang T-L. PopLDdecay: a fast and effective tool for linkage disequilibrium decay analysis based on variant call format files. Bioinformatics. 2019;35(10):1786-8. https://doi.org/10.1093/ bioinformatics/bty875.
55. Raj A, Stephens M, Pritchard JK. fastSTRUCTURE: Variational inference of population structure in large SNP data sets. Genetics. 2014;197(2):573-89. https://doi.org/10.1534/genetics.114.164350.

56. Cingolani $P$, Platts $A$, Wang $L L$, Coon $M$, Nguyen $T$, Wang $L$, et al. A program for annotating and predicting the effects of single nucleotide polymorphisms, SnpEff: SNPs in the genome of Drosophila melanogaster strain w1118; iso-2; iso-3. Fly. 2012;6(2):80-92. https://doi. org/10.4161/fly. 19695

\section{Publisher's Note}

Springer Nature remains neutral with regard to jurisdictional claims in published maps and institutional affiliations.
Ready to submit your research? Choose BMC and benefit from:

- fast, convenient online submission

- thorough peer review by experienced researchers in your field

- rapid publication on acceptance

- support for research data, including large and complex data types

- gold Open Access which fosters wider collaboration and increased citations

- maximum visibility for your research: over $100 \mathrm{M}$ website views per year

At BMC, research is always in progress.

Learn more biomedcentral.com/submissions 